\title{
ESTADO DE SAÚDE DE PACIENTES ACOMETIDOS COM A DOENÇA DE ALZHEIMER EM UM HOSPITAL PÚBLICO DO DISTRITO FEDERAL
}

\author{
HEALTH STATUS OF PATIENTS WITH ALZHEIMER'S DISEASE \\ IN A PUBLIC HOSPITAL IN FEDERAL DISTRICT \\ Maria Célia Fonseca Magalhães ${ }^{{ }^{*}}$, Renata Costa Fortes ${ }^{b^{*}}$ \\ acelya_fm@hotmail.com, bfortes.rc@gmail.com \\ *Universidade Paulista, Brasília (DF), Brasil
}

\section{RESUMO}

Introdução: Atualmente, a população mundial vem aumentando sua longevidade, o que representa um incremento no número de idosos. Uma das maiores preocupações em relação à saúde psicológica dos idosos é o avanço da doença conhecida como Alzheimer. Objetivo: Analisar o estado de saúde dos pacientes acometidos com doença de Alzheimer. Método: Estudo retrospectivo e descritivo realizado no Hospital Regional da Asa Norte (HRAN) no período entre agosto e novembro de 2012. A coleta de dados foi realizada por meio de registros clínicos do Núcleo de Marcação de Consultas e Arquivo Médico do HRAN de pacientes idosos com diagnóstico de doença de Alzheimer, atendidos entre abril de 2011 e abril de 2012. Utilizou-se a ficha de avaliação nutricional Mini Avaliação Nutricional (MAN) e fichas de avaliação neuropsicológica como a avaliação cognitiva Mini-Mental State de Folstein (MMS), assim como a ficha de análise da depressão geriátrica (EDG) e o Clinical Dementia Rating (CDR), "CDR de Hughes". Resultados: Observou-se, após análise de 80 prontuários, que 2,50\% $(\mathrm{n}=4)$ dos idosos estavam desnutridos e 18,75\% $(\mathrm{n}=12)$ em risco de desnutrição; $13,75 \%(\mathrm{n}=11)$ com demência e 7,50\% $(n=6)$ com prejuízo cognitivo causado por depressão; $27,50 \%(n=22)$ déficit da capacidade cognitiva leve e 5\% $(n=4)$ moderada. Conclusão: Os resultados apontam elevada presença de desnutrição (ou risco nutricional), demência, prejuízo cognitivo e depressão leve a moderada em idosos com doença de Alzheimer. Porém, cabe destacar que a falta de alguns registros dos dados nos prontuários constitui uma das limitações do presente estudo.

Palavras-chave: Doença de Alzheimer; saúde mental; envelhecimento.

\section{ABSTRACT}

Introduction: Currently, the world's population is increasing its longevity, which represents an increase in the level of the elderly population. One of the biggest concerns about the psychological health of the elderly is the progression of the disease known as Alzheimer's. Objective: To assess the health status of patients suffering with Alzheimer's disease. Method: Retrospective descriptive study conducted at Hospital Regional da Asa Norte (HRAN) from August of 2012 to November of the same year. Data collection was performed by clinical records of the Appointment Booking and Medical Archive HRAN of elderly patients diagnosed with Alzheimer's disease, treated between April 2011 and April 2012. We used nutritional assessment sheets Mini Nutritional Assessment (MNA) and neuropsychological assessment sheets as the cognitive assessment Mini-Mental State (MMS) of Folstein as well as the form of the Geriatric Depression (EDG) analysis and Clinical Dementia Rating (CDR), "CDR Hughes ". Results: It was observed, after analyzing eighty reports, that $2.50 \%(\mathrm{n}=4)$ of the elderly were malnourished and $18.75 \%(n=12)$ were at risk of undernourishment; $13,75 \%(n=11)$ 
of the elderly had dementia and $7.50 \%(n=6)$ with cognitive impairment due to depression. Conclusion: The results point out that undernourishment (or the risk of it), dementia, cognitive impairment and light or moderated depression. However, it must be said that the absence of some information on the reports made this study more limited.

Keywords: Alzheimer's disease; mental health; aging.

\section{Introdução}

A sociedade contemporânea passa por uma transição demográfica, evidenciada por um crescimento da população idosa. O envelhecimento do homem é um processo dinâmico e progressivo, com alteraçóes morfológicas, fisiológicas e psicológicas. Isso tem acarretado um aumento da prevalência de doenças crônicas, que necessitam de tratamentos complexos e de longa duração. Dentre estas, destacamos as doenças cardiovasculares $(\mathrm{DCV})$, que respondem pelo maior índice dos óbitos no país, elevando as despesas com assistência hospitalar no SUS e totalizando por volta de $75 \%$ dos gastos com atenção à saúde ${ }^{1,2}$.

Dentre os principais fatores de risco para DCV, a hipertensão arterial (HA) e o diabetes mellitus (DM) configuram importantes problemas, sendo as principais causas de morbimortalidade na população brasileira. Ambas são condiçóes crônicas que possuem elevadas taxas de prevalências, acarretando complicaçóes agudas e crônicas. Frequentemente acometem mais os idosos, lembrando que a incidência aumenta com a idade $e^{3,4,5,6}$.

A prevalência de HA em pacientes diabéticos é pelo menos duas vezes a da população em geral $^{2,3,7}$. Nesse sentido, diversos estudos sobre HA e DM têm mostrado que o controle rigoroso da glicemia e da pressão arterial é capaz de reduzir as complicaçóes e os tratamentos dos problemas crônicos de saúde tem como finalidade reduzir a morbimortalidade e manter a qualidade de vida das pessoas enfermas ${ }^{3,4,5,8}$.

Para tal, na HA utilizam-se medidas não medicamentosas isoladas ou associadas a medicamentos anti-hipertensivos. Várias abordagens no tratamento resultam na redução dos níveis de pressão arterial e provocam também alteraçóes na qualidade de vida do paciente 5 .

Já para a terapêutica do DM, recomenda-se, inicialmente, o uso de medidas não farmacológicas. Se as medidas iniciais não forem suficientes, indica-se o uso de medicamentos, monoterapia oral ou combinação oral com medicamentos que tenham ações farmacológicas diferentes. Quando não se obtém o controle, inicia-se insulinoterapia isolada ou associada à medicação oral. As medidas não farmacológicas incluem mudanças nos hábitos alimentares, através de orientação médica ou de nutricionistas, e atividade física orientada ${ }^{10,11}$.

A modificação no estilo de vida, como é denominada a terapia não farmacológica, inclui a redução do peso corporal, da ingestão do sal e gorduras, do consumo de bebidas alcoólicas, o fim do tabagismo e a prática de exercícios físicos com regularidade. Elas são indicadas tanto para um tratamento inicial quanto para associação com a terapia farmacológica. De baixo custo e risco mínimo, atuam no aumento da eficácia do tratamento medicamentoso e no controle de outros fatores de risco cardiovasculares ${ }^{1}$.

Assim, vários fatores podem influenciar a adesão ao tratamento, tais como idade, gênero, etnia, estado civil, escolaridade, nível socioeconômico, autoestima, crenças de saúde, hábitos de vida e culturais, cronicidade da doença, ausência de sintomas e consequências tardias decorrentes da doença, custo do tratamento, efeitos indesejáveis do tratamento, esquemas terapêuticos complexos, o acesso ao serviço de saúde e às políticas de saúde, e, finalmente, o relacionamento com a equipe de saúde ${ }^{9,12}$.

O desenvolvimento de pesquisas que buscam entender os aspectos que levam o idoso a não adesão (ou adesão inadequada) do tratamento pode contribuir para a elaboração de intervençôes mais eficazes em educação em saúde para esse grupo etário, contribuindo para a redução dos altos índices de internação, morbidade e mortalidade, além de melhorar a qualidade de vida deles. A partir disso, este estudo tem como objetivo avaliar o grau de adesão ao regime terapêutico (farmacológico e não farmacológico) de idosos com hipertensão e/ou diabetes internados em um hospital universitário.

\section{Método}

Trata-se de um estudo descritivo de corte transversal, realizado em um hospital de ensino e público que presta serviços de baixa, média e alta complexidade, referência na cidade de Cuiabá, no Estado de Mato Grosso.

Participaram do estudo 30 idosos com idade igual ou superior a 60 anos de ambos os sexos, com diagnóstico de HA e/ou DM, internados no período de julho de 2011 a janeiro de 2012 nas clínicas médica e cirúrgica. Foram considerados como critérios de inclusão deste estudo: ter 60 anos ou mais; ter o diagnóstico de HA e/ou de DM; estar internado na instituição, não apresentar confusão mental, compreender e concordar com os objetivos e metodologia do estudo e assinar o TCLE. 
A aplicação do questionário foi realizada diretamente com o paciente, na própria clínica. Os dados foram coletados por meio de um questionário próprio, que teve como referência para elaboração, a Caderneta de Saúde da Pessoa Idosa $^{13}$ e o formulário utilizado para o cadastramento de hipertensos e diabéticos no programa Hiperdia ${ }^{14}$.

Utilizou-se um questionário que contemplava informaçóes sócio-demográficas (sexo, idade, grau de escolaridade, renda familiar, com quem mora, estado civil e ocupação) e dados sobre as condições de saúde (histórico familiar de HA e DM, diagnóstico, tempo de diagnóstico, uso de bebida alcoólica, uso de tabaco, prática de atividade física, complicaçôes referentes à HA e DM, internaçóes, procura às unidades de saúde para verificar pressão arterial e/ ou glicemia e realizar automedida em sua residência da pressão arterial e/ou glicemia). Além disso, dados relativos à terapêutica, como o tipo de tratamento, medicamentos que utilizava, quantos comprimidos utilizava diariamente e orientaçóes de medidas não farmacológicas (dieta apropriada, prática de atividade física, diminuiçãa da ingestão de sal e de bebida alcoólica) e o grau de adesão ao tratamento segundo o teste de Morisky ${ }^{15}$.

Para identificar o grau de adesão ao tratamento medicamentoso, utilizou-se o teste de Morisky ${ }^{13}$, que é composto por quatro perguntas que buscam avaliar o comportamento do paciente em relação ao uso habitual do medicamento. Considera-se alto grau de adesão quando todas as respostas são negativas. Porém, quando pelo menos uma das respostas é afirmativa, o paciente é classificado no grupo de baixo grau de adesão.

Os dados foram digitados e analisados no programa SPSS 14.0, onde foram realizadas análises descritivas e inferências para verificar a correlação entre as variáveis.

Este estudo foi submetido à apreciação e teve a aprovação do comitê de ética e pesquisa do Hospital Universitário Julio Muller, parecer No 63/2011. Todos os sujeitos que concordaram em participar da pesquisa fizeram a leitura do Termo de Consentimento Livre e Esclarecido (TCLE) e, subsequente, a assinatura do mesmo.

\section{Resultados}

As características demográficas e sociais dos 30 idosos incluídos no estudo, hipertensos e/ou diabéticos, internados na clínica médica e clínica cirúrgica, são apresentadas na Tabela 1 . Houve predomínio do sexo masculino $19(63,3 \%), 46,7 \%(n=21)$ com faixa etária entre 65 e 75 anos, 50,0\% ( $n=15)$ casados e com baixo grau de escolaridade $(\mathrm{N}=11 ; 36,7 \%)$ com 1 a 4 anos de estudo. Quanto à renda, 73,3\% $(n=22)$ recebem um salário mínimo e $70 \%(\mathrm{n}=21)$ eram aposentados.
Tabela 1: Distribuição dos idosos dos idosos hipertensos e/ou diabéticos internados na clínica médica e cirúrgica segundo características sóciodemográficas. Cuiabá (MT), 2012

\begin{tabular}{|c|c|c|}
\hline Variáveis & $\mathrm{n}$ & $\%$ \\
\hline \multicolumn{3}{|l|}{ Sexo } \\
\hline Feminino & 11 & 36,7 \\
\hline Masculino & 19 & 63,3 \\
\hline \multicolumn{3}{|l|}{ Idade } \\
\hline 60 a 64 anos & 9 & 30,0 \\
\hline 65 a 75 anos & 14 & 46,7 \\
\hline 76 anos ou mais & 7 & 23,3 \\
\hline \multicolumn{3}{|l|}{ Estado civil } \\
\hline Casado & 15 & 50,0 \\
\hline Solteiro & 4 & 13,3 \\
\hline Viúvo & 9 & 30,0 \\
\hline Separado & 2 & 6,7 \\
\hline \multicolumn{3}{|l|}{ Grau de escolaridade } \\
\hline Analfabeto & 10 & 33,3 \\
\hline 1 a 4 anos & 11 & 36,7 \\
\hline 5 a 8 anos & 4 & 13,3 \\
\hline Mais de 8 anos & 5 & 16,7 \\
\hline \multicolumn{3}{|l|}{ Ocupação } \\
\hline Aposentado & 21 & 70,0 \\
\hline Autônomo & 4 & 13,3 \\
\hline $\begin{array}{l}\text { Empregado com cartei- } \\
\text { ra assinada }\end{array}$ & 2 & 6,7 \\
\hline Do lar & 3 & 10,0 \\
\hline \multicolumn{3}{|l|}{ Renda familiar } \\
\hline Sem renda & 2 & 6,7 \\
\hline 1 salário mínimo & 22 & 73,3 \\
\hline 2 a 4 salários mínimos & 6 & 20,0 \\
\hline Total & 30 & 100,0 \\
\hline
\end{tabular}

A Tabela 2 apresenta os dados de saúde e doença dos idosos, que revela que $21(70,0 \%)$ são hipertensos e 7 $(23,3 \%)$ hipertensos e diabéticos. A maioria afirmou saber do diagnóstico por mais de seis anos, e dos idosos diabéticos $(n=20 ; 66,7 \%)$. Em relação às complicaçóes dessas morbidades, $16,7 \%(n=5)$ foram acometidos por Acidente Vascular Encefálico (AVE). No que se refere às características do tratamento, houve predomínio do tratamento medicamentoso $(n=27 ; 90,0 \%)$. E a quantidade de comprimidos ingeridos pelos idosos diariamente para o controle da pressão arterial e dos níveis de glicemia foi de 40,0\% ( $n=12)$ quatro ou mais de seis comprimidos.

Quanto ao grau de adesão ao tratamento medicamentoso, segundo o teste de Morisky, observou que: 24 $(82,7 \%)$ apresentaram baixo grau de adesão ao tratamento medicamentoso e somente $5(17,2 \%)$ apresentaram alto grau de adesão ao tratamento medicamentoso, conforme a Tabela 3 . 
Tabela 2: Distribuição dos idosos dos idosos hipertensos e/ou diabéticos internados na clínica médica e cirúrgica segundo condições de saúde. Cuiabá (MT), 2012

\begin{tabular}{|c|c|c|}
\hline Variáveis & $\mathbf{n}$ & $\%$ \\
\hline \multicolumn{3}{|l|}{ Patologia } \\
\hline $\begin{array}{l}\text { Hipertensão } \\
\text { arterial }\end{array}$ & 21 & 70,0 \\
\hline Diabetes mellitus I & 1 & 3,3 \\
\hline $\begin{array}{l}\text { Diabetes } \\
\text { mellitus II }\end{array}$ & 1 & 3,3 \\
\hline $\begin{array}{l}\text { Hipertensão } \\
\text { arterial e diabetes } \\
\text { mellitus }\end{array}$ & 7 & 23,3 \\
\hline \multicolumn{3}{|l|}{ Histórico familiar } \\
\hline $\begin{array}{l}\text { Hipertensão } \\
\text { arterial }\end{array}$ & 14 & 46,7 \\
\hline Diabetes mellitus & 3 & 10,0 \\
\hline \multicolumn{3}{|c|}{ Tempo de diagnóstico } \\
\hline \multicolumn{3}{|l|}{ Hipertensão arterial } \\
\hline $\begin{array}{l}\text { Menos de } 1 \text { ano e } \\
11 \text { meses }\end{array}$ & 4 & 13,3 \\
\hline $\begin{array}{l}\text { de } 4 \text { a } 5 \text { anos e } 11 \\
\text { meses }\end{array}$ & 2 & 6,7 \\
\hline Mais de 6 anos & 20 & 66,7 \\
\hline \multicolumn{3}{|l|}{ Diabetes mellitus } \\
\hline $\begin{array}{l}4 \text { a } 5 \text { anos e } 11 \\
\text { meses }\end{array}$ & 5 & 16,7 \\
\hline Mais de 6 anos & 3 & 10,0 \\
\hline \multicolumn{3}{|l|}{ Complicaçôes } \\
\hline $\begin{array}{l}\text { Acidente Vascular } \\
\text { Encefálico (AVE) }\end{array}$ & 5 & 16,7 \\
\hline $\begin{array}{l}\text { Infarto Agudo do } \\
\text { Miocárdio (IAM) }\end{array}$ & 2 & 6,6 \\
\hline AVE e IAM & 2 & 6,7 \\
\hline \multicolumn{3}{|l|}{ Tratamento } \\
\hline Medicamentoso & 27 & 90,0 \\
\hline $\begin{array}{l}\text { Medicamentoso e } \\
\text { fitoterápico }\end{array}$ & 2 & 6,7 \\
\hline $\begin{array}{l}\text { Não } \\
\text { medicamentoso }\end{array}$ & 1 & 3,3 \\
\hline \multicolumn{3}{|c|}{ Quantos comprimidos diariamente? } \\
\hline Apenas 1 & 4 & 13,3 \\
\hline 2 a 3 & 8 & 26,7 \\
\hline 4 a 5 & 8 & 26,7 \\
\hline $\begin{array}{l}6 \text { ou mais } \\
\text { Total }\end{array}$ & $\begin{array}{l}10 \\
30\end{array}$ & $\begin{array}{c}33,3 \\
100,0 \\
\end{array}$ \\
\hline
\end{tabular}

Tabela 3: Distribuição dos idosos dos idosos hipertensos e/ou diabéticos internados na clínica médica e cirúrgica segundo o grau de adesão ao tratamento medicamentoso. Cuiabá (MT), 2012

\begin{tabular}{lcc}
\hline Variáveis & N & $\%$ \\
Alto grau de adesão & 5 & 17,2 \\
$\begin{array}{l}\text { Baixo grau de } \\
\text { adesão }\end{array}$ & 24 & 82,7 \\
Total $^{*}$ & 29 & 100,0 \\
\hline
\end{tabular}

$\mathrm{N}=29$, pois um idoso nâo realiza tratamento medicamentoso
Quanto ao cumprimento das orientaçóes de medidas não farmacológicas dadas pela equipe de saúde, observou-se que $23(76,7 \%)$ dos idosos fazem dieta apropriada; $21(70,0 \%)$ não praticam nenhuma atividade física; $18(60 \%)$ seguem a orientação de baixa ingestão de sódio na dieta, e quanto à redução do consumo de bebida alcoólica, $22(73,3 \%)$ dos idosos seguem esta orientação. Observamos também que 26 $(86,7 \%)$ dos idosos deste estudo nem sempre seguem as orientaçóes não farmacológicas e apenas $4(13,3 \%)$ afirmam que sempre seguem as orientaçôes não farmacológicas, conforme apresentados na Tabela 4.

Tabela 4: Distribuição dos idosos dos idosos hipertensos e/ou diabéticos internados na clínica médica e cirúrgica segundo medidas não farmacológicas. Cuiabá (MT), 2012

\begin{tabular}{lcc}
\hline Variáveis & n & $\%$ \\
$\begin{array}{l}\text { Orientação de medidas não } \\
\text { farmacológicas }\end{array}$ & \\
$\begin{array}{l}\text { Sempre seguem as orientações não } \\
\text { farmacológicas }\end{array}$ & 4 & 13,3 \\
$\begin{array}{l}\text { Nem sempre seguem as orientações } \\
\text { não farmacológicas }\end{array}$ & 26 & 86,7 \\
Total & 30 & 100,0 \\
\hline
\end{tabular}

\section{Discussão}

Neste estudo, observou-se a prevalência de idosos portadores de HA e DM do gênero masculino, com a faixa etária entre 65 e 75 anos. Provavelmente, a maioria da população é formada por homens pelo fato de serem a maioria entre os idosos internados ${ }^{16}$. Além disso, indicadores demográficos populacionais, relacionados ao gênero, apontam que os idosos do sexo masculino apresentam maiores riscos de adoecer e morrer quando comparados aos do sexo feminino ${ }^{17}$.

Quanto à escolaridade, houve predomínio da baixa escolaridade. O baixo nível de escolaridade pode ser consequência do pouco acesso à escola quando crianças. Esse dado apresenta-se como um desafio para os profissionais, que devem abordar as questôes de saúde e as intervençôes de uma maneira compreensível, de acordo com as necessidades de cada indivíduo ${ }^{17}$.

Quanto ao tipo de morbidade, observou-se que mais da metade dos idosos eram hipertensos. Isso acontece pelo fato da HA variar de 50 a $70 \%$ na população geriátrica, aumentando linearmente com a idade ${ }^{9}$. Nos diabéticos a hipertensão arterial é duas vezes mais frequente do que na população em geral ${ }^{18}$. Esta situação é incrementada pela ausência de atividade física e dieta inadequada, tornando um grave fator de risco para ocorrências de doenças cardiovasculares ${ }^{19}$. 
Para tanto, a abordagem da hipertensão arterial e da diabetes é constituída de intervenção farmacológica e não farmacológica sempre acompanhadas por mudanças no estilo de vida. Neste sentido, o sucesso do controle das doenças crônicas depende, sobretudo, da adesấo adequada do paciente ao tratamento e às práticas de saúde que estimulem ou facilitem a mudança do estilo de vida ${ }^{4}$.

Assim, a adesão ao tratamento em pacientes crônicos representa a extensão em que o comportamento da pessoa coincide com o aconselhamento dado pelo profissional de saúde. Trata-se de um processo dinâmico, influenciado por vários fatores, que requer um acompanhamento cuidadoso, com intervenções específicas em termos individuais em que a família, as organizaçóes e a comunidade em geral têm um papel importantíssimo ${ }^{20}$.

Nossos achados por meio do teste de Morisky mostraram que mais da metade dos idosos com hipertensão arterial e/ou diabetes apresentavam comportamento indicativo de baixo grau de adesão, sendo os idosos considerados muito vulneráveis quanto a adesão ${ }^{21}$. Os resultados corroboram com um estudo realizado com idosos hipertensos atendidos em um centro de saúde de Novo Horizonte, São Paulo, Brasil, que avaliou a adesão pelo teste de Morisky, onde apenas $28 \%$ dos indivíduos tiveram adesão ao tratamento ${ }^{22}$. Entretanto, uma pesquisa realizada em Santa Catarina em 2012, que avaliou a adesáo aos medicamentos em idosos hipertensos pelo TMG, apresentou maior prevalência de adesão ao tratamento ${ }^{23}$.

A baixa adesão é complexa e multifatorial, associado a distintos fatores, como os relacionados ao doente, os sociodemográficos, os culturais e os comportamentais, os relacionados aos medicamentos e aos vários aspectos clínicos, os ambientais e os econômicos ${ }^{24}$.

Neste contexto, temos que ressaltar o fator relacionado ao uso de medicações, visto que, os estudos apontam que $80 \%$ dos idosos fazem uso de algum medicamento. Assim, o uso racional destes, trouxe muitos ganhos para a saúde nas últimas décadas, como os medicamentos que controlam a hipertensão arterial e o diabetes, que contribuem para a prevenção de Acidentes Vasculares Cerebrais e Infarto do Miocárdio e, consequentemente, evitam muitas mortes prematuras. No entanto, a utilização dos fármacos requer cuidados específicos para evitar interaçóes, reaçóes adversas, controlar efeitos secundários para aumentar a qualidade de vida e acarretar em uma diminuiçấo da morbilidade e da mortalidade ${ }^{21}$.

Quanto a aderir medidas não farmacológicas, a maioria dos idosos confirmou que nem sempre segue as orientaçôes. Em um estudo realizado no hospital universitário do Rio Grande do Sul observou-se também que poucos idosos eram aderentes ao tratamento não farmacológico $(18 \%)^{25}$. Assim, as recomendaçōes de mudanças no estilo de vida relacionadas ao tratamento não farmacológico apresentam geralmente percentuais mais elevados de não adesão quando comparadas ao tratamento farmacológico, apesar de diversas pesquisas comprovarem que a "adesáo" a essas orientaçôes podem impactar significativamente os sintomas, a capacidade funcional, o bem-estar, a morbidade e o prognóstico da doença ${ }^{21}$.

A adesão terapêutica requer uma abordagem multidisciplinar com intervençóes concentradas em todos os intervenientes no processo de cuidar. As estratégias de intervenção têm como objetivo melhorar a adesão dos doentes ou promover o desenvolvimento de competências dos profissionais para aumentar a adesão de quem cuidam. Essas intervençóes podem ser classificadas em educacionais e comportamentais. As educacionais incluem o fornecimento de informação escrita ou oral e material audiovisual (pelo médico, enfermeiro, farmacêutico, dentre outros) individualmente ou em grupo. Já as intervenções comportamentais contribuem para aumentar a comunicaçâo e aconselhamento, simplificar os regimes terapêuticos. Apesar de essas intervençóes estarem identificadas, ainda não se conhece bem a sua eficácia ${ }^{3,7,21}$.

É grande a repercussão da não adesão a regimes terapêuticos entre idosos ${ }^{1}$, tornando esse um dos grandes problemas de saúde no mundo em face da elevada prevalência de doenças crônicas e sendo responsável pelo aumento da probabilidade do insucesso da terapêutica por complicaçóes evitáveis, por aumento dos gastos com a saúde, aumento da morbidade e mortalidade ${ }^{26}$.

\section{Conclusão}

Foi alta a não adesão ao tratamento medicamentoso e não farmacológico dos idosos internados na clínica médica e cirúrgica. Neste estudo também foram analisados possíveis aspectos que afetam a adesáo ao tratamento, como os sócio-demográficos e os relacionados à doença e à terapêutica. Porém, destaca-se a necessidade de avaliar, além desses fatores, os relacionados aos profissionais e ao sistema de saúde, gerando, assim, uma maior atenção para aspectos da atenção à saúde, como a comunicaçáo, a preocupação no investimento na informação (educação em saúde), multidisciplinaridade e também a atualização dos profissionais de saúde. Faz-se necessária uma discussão entre equipe multidisciplinar de saúde, idosos, familiares e gestores sobre o problema da não adesão ao tratamento, bem como possibilidades para resolução, pois a não adesão compromete a qualidade de vida do idoso. 


\section{Agradecimento}

Ao hospital, pelo auxílio na operacionalização deste estudo.

\section{Referências}

1. Almeida HO, Versiani ER, Dias AR, Novaes MRCG, Trindade, EMV. Adesão a tratamentos entre idosos. Comun Ciênc Saúde. 2007;18(1):57-67.

2. Brasil. Ministério da Saúde. Secretaria de Atenção à Saúde. Departamento de Açôes Programáticas e Estratégicas. Atenção à saúde da pessoa idosa e envelhecimento/ Ministério da Saúde, Secretaria de Atenção à Saúde, Departamento de Ações Programáticas e Estratégicas, Área Técnica Saúde do Idoso. Brasília, 2010.

3. Santos PA, Pinho CPS. Diabetes mellitus em pacientes coronariopatas: prevalência e fatores de risco cardiovascular associado. Rev Bras Clin Med. 2012;10(6):469-75.

4. Silva TR, Feldmam C, Lima MHA, Nobre MRC, Domingues RZL. Controle de diabetes mellitus e hipertensão arterial com grupos de intervenção educacional e terapêutica em seguimento ambulatorial de uma Unidade Básica de Saúde. Saude Soc. 2006;15(3):180-189.

5. Monteiro LZ, Fiani CRV, Freitas MCF, Zanetti ML, Foss MC. Redução da pressão arterial, da IMC e da glicose após treinamento aeróbico em idosas com diabete tipo 2. Arq Bras Cardiol. 2010;95(5):563-570.

6. Martins LN, Souza LS, Silva CF, Machado RS; Silva CEF, Vilagra MC, et al. Prevalência dos fatores de risco cardiovascular em adultos admitidos na unidade de dor torácica em Vassouras, RJ. Rev Bras Cardiol. 2011;24(5):299-307.

7. Knuth AG, Bielemann RM, Silva SG, Borges TT, Del Duca GF, Kremer MM, et al. Conhecimento de adultos sobre o papel da atividade física na prevenção e tratamento de diabetes e hipertensão: estudo de base populacional no Sul do Brasil. Cad. Saúde Pública. 2009;25(3):513-520.

8. Reiners AAO, Azevedo RCS, Vieira MA, Arruda ALG. Produção bibliográfica sobre adesão/não adesão de pessoas ao tratamento de saúde. Ciênc Saúde Coletiva. 2008;13(Suppl 2):2299-2306.

9. Vitor RS, Sakai FK, Consoni, PRC. Indicaçáo e adesão de medidas não farmacológicas no tratamento da hipertensão arterial. Rev AMRIGS. 2009;53(2):117-121.

10. Santos FS, Oliveira KR, Colet CF. Adesão ao tratamento medicamentoso pelos portadores de Diabetes Mellitus atendidos em uma Unidade Básica de Saúde no município de Ijuí/RS: um estudo exploratório. Rev Ciênc Farm Básica Apl. 2010;31(3):223-227.

11. Espírito Santo MB, Souza LME, Souza ACG, Ferreira FM, Silva CNMR, Taitson PF. Adesão dos portadores de diabetes mellitus ao tratamento farmacológico e não farmacológico na atenção primária à saúde. Enf Rev. 2012;15(1):88-101.

12. Rocha CH, Oliveira APS, Ferreira C, Faggiani FT, Schroeter G, de Souza ACA, et al. Adesão à prescrição médica em idosos de Porto Alegre, RS. Ciênc. Saúde Colet. 2008;13(Suppl):703-710.

13. Brasil. Ministério da Saúde. Caderneta de saúde da pessoa Idosa. Brasília: Ministério da Saúde; 2007.

14. Brasil, Ministério da Saúde. Plano de reorganização da atenção à hipertensão arterial e ao diabetes mellitus. Manual de hipertensão arterial e diabetes mellitus. Brasília: Ministério da Saúde; 2002.

15. Morisky DE, Green LW, Levine DM. Concurrent and predictive validity of a self-reported measure of medication adherence. Med Care. 1986;24(1):67-74.

16. Brasil. Ministério da Saúde. DATASUS. Morbidade Hospitalar do SUS. Disponível em: http://www2.datasus. gov.br/DATASUS/index.php? area=0205\&VObj=http:// tabnet.datasus.gov.br/cgi/ deftohtm.exe?sim/cnv/obt10. Acesso em julho de 2012.

17. Santos MIPO. Perfil dos idosos internados no Hospital Geral em Belém (Pará). Esc. Anna Nery. 2007;11(1):23-29.

18. Spinel LF, Püschel VAA. Perfil de estilo de vida de pessoas com doença cardiovascular. Rev Gaúcha Enferm. 2007;28(4):534-41.

19. Molina MCB, Cunha RS, Herkenhoff LF, Mill JG. Hipertensão arterial e consumo de sal em população urbana. Rev Saúde Pública. 2003;37(6):743-750.

20. Pereira JC, Barreto SM, Passos VMA. O perfil de saúde cardiovascular dos idosos brasileiros precisa melhorar: estudo de base populacional. Arq Bras Cardiol. 2008;91(1):1-10.

21. Henriques MAP. Adesão ao regime medicamentoso em idosos na comunidade: eficácia das intervençôes de enfermagem [tese]. Lisboa: Universidade de Lisboa; 2006.

22. Eid LP, Nogueira MS, Veiga EV, Cesarino EJ, Alves LMM. Adesão ao tratamento anti-hipertensivo: análise pelo Teste de Morisky-Green. Rev Eletr Enf. 2013;15(2):362-7

23. Pucci N, Pereira MR, Vinholes DB, Pucci P, Campos ND. Conhecimento sobre hipertensao arterial sistêmica e adesão ao tratamento anti-hipertensivo em idosos. Rev Bras Cardiol. 2012;25(4):322-9

24. Castro RA, Aliti GB, Linhares JC, Rabelo ER. Adesão ao tratamento de pacientes com insuficiência cardíaca em um hospital universitário. Rev Gaúcha Enferm. 2010;31(2):225-31.

25. Santos ZMSA, Frota MA, Cruz DM, Olanda SDO. Adesão do cliente hipertenso ao tratamento: análise com abordagem interdisciplinar. Texto Contexto Enferm. 2005 jul./ set;14 (3): 332-40.

26. Dewulf NLS, Monteiro RA, Passos ADC, Vieira EM, Troncon LEA. Adesão ao tratamento medicamentoso em pacientes com doenças gastrintestinais crônicas acompanhados no ambulatório de um hospital universitario. Rev Bras Ciênc Farm. 2006;42(4):575-584. 